\title{
Non-linear optical properties and absolute structure of metastable 4-methyl benzophenone
}

\author{
W. Kaminsky ${ }^{1}$, E. Weckert ${ }^{2}$, H. Kutzke ${ }^{3}$, M. A. \\ Glazer $^{4}$, H. Klapper ${ }^{3}$ \\ 1: Department of Chemistry, University of Washington, \\ Seattle, WA 98196, USA \\ 2. HASYLAB at DESY, Notkestr. 85, D-22603 Hamburg, \\ Germany \\ 3: Mineralogisch-Petrologisches Institut, Universitaet Bonn, \\ D-53115 Bonn, Germany \\ 4: Clarendon Laboratory, University of Oxford, Parks Road, \\ OX1 3PU, Oxford, England
}

\section{Received; accepted}

\begin{abstract}
Metastable 4-methyl benzophenone (4-MBP, $\mathrm{C}_{14} \mathrm{H}_{11} \mathrm{O}$ ) crystallizes in the polar (pyroelectric) and enantiomorphic space groups (SG) $P 3_{1}$ and $P 3_{2}$. The optical rotation tensor has been measured for $670 \mathrm{~nm}$ and the material was tested for evidence of phase-matchability. The absolute structure was determined via three-beam diffraction, showing that the structural right-hand screw $3_{1}$ is combined with positive (right-handed, i.e. clockwise when looking towards the light source) optical rotation, for both tensor components $\rho_{11}=\rho_{22}=26(5)^{\circ} / \mathrm{mm}, \rho_{33}=$ $8.3(5)^{\circ} / \mathrm{mm}$ (contrary to low-quartz which shows negative optical rotation for $\rho_{33}$ and positive rotation for $\rho_{11}$ in righthand SP $P 3_{1} 2$ ).

The non-linear optical tensors for optical rotation, $d$ coefficients, electro-optic effect and electrogyration have been calculated using a set of electronic polarizabilities as input parameters for a dipole-dipole interaction model that reproduces the experimental refractive indices and optical rotation. Unusually high polarizability volumes are needed for hydrogen atom bonding to aromatic carbons in order to model the optical tensors. However, the non-linear optical coefficients are of average size.
\end{abstract}

\section{Introduction}

4-Methyl benzophenone (4-MBP, $\mathrm{C}_{14} \mathrm{H}_{11} \mathrm{O}$ ) crystallizes in two different polymorphic forms: a stable phase (melting point [m.p.] $58-59^{\circ} \mathrm{C}$ ) with centrosymmetric monoclinic space group $P 2{ }_{1} / c$, and a metastable phase (m.p. $54-55^{\circ} \mathrm{C}$ ) 
with polar (pyroelectric) and enantionmorphic space groups $P 3_{1}$ and $P 3_{2}[1,2,3]$. The metastable phase can only be nucleated from highly supercooled melt $\left(<-20^{\circ} \mathrm{C}\right)[3,4]$, whereas the stable crystals can be easily precipitated from solutions and from (moderately) supercooled melts. However, large metastable crystals of sufficiently good perfection can be grown from slightly supercooled melt by using a metastable seed crystal $[3,5]$. The typical polar growth morphology of these crystals, together with their pyroelectric polarity, is shown in Figure 1. The crystal structure has been determined by single-crystal X-ray diffraction [3]. Metastable 4-MBP tends to transform into the stable phase, in particular when coming into contact with stable-phase particles. Once initiated, the monotropic transformation proceeds irresistibly within several minutes to an hour through a crystal of up to $\mathrm{a} \mathrm{cm}^{3}$ in size, leaving behind a milky polycrystalline aggregate of stable 4-MBP.

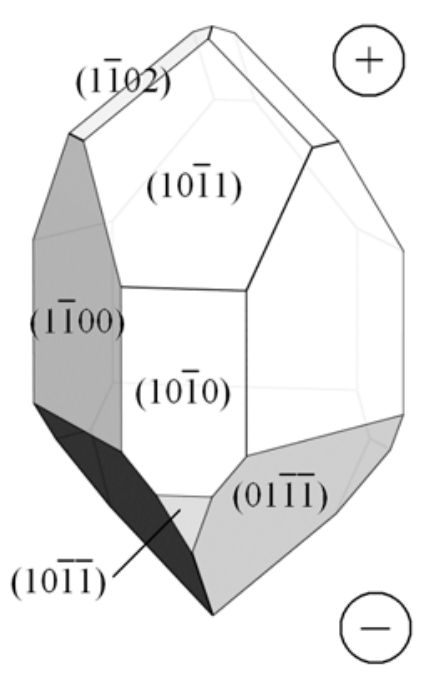

Fig. 1. Typical morphology of metastable 4-MBP crystals grown from supercooled melt, with face forms $\{1 \overline{0} 0\},\{\overline{1} 00\}$ (trigonal prisms) and $\{1 \overline{1} 1\},\{\overline{1} 02\}$ (small), $\{0 \overline{1}\}$ (trigonal pyramids) (graphics program: WinXMorph [6]). Polar trigonal axis vertical. The plus and minus sign indicate the signs of the pyroelectric charges developing during heating $[1,2]$. The same signs have the piezoelectric charges generated by compressional stress $-\sigma_{33}$ along the polar axis [7].

Despite being chemically identical, stable and metastable 4MBP exhibit complete different packing arrangements. This is connected with a striking change in the dihedral angles and a slight change in the bond angles of the carbonyl group. Hence, the two polymorphs of 4-MBP exhibit considerable differences in their physical features. A comparative study of even-rank tensor properties of both 4MBP polymorphs (thermal expansion, elasticity and thermoelasticity) show that the differences are small but nevertheless significant [7]. The essential differences, however, appear in the odd-rank polar tensor properties, which are zero in centrosymmetric stable 4-MBP, but exist in acentric metastable 4-MBP: the pyroelectric $\pi_{33}$ was 
found to be quite strong, comparable to that of tourmaline, whereas the piezoelectric effect is moderate with $d_{111}$ about the same as in quartz and $d_{333}$ about 6 times smaller than $d_{111}$ [7]. Second harmonic generation (SHG) has not been studied in 4-MBP as far as we know.

In addition, optical rotation (optical activity, OR) is presented here as an example of even-rank axial properties which exist only in the metastable polymorph of 4-MBP.

The question arises what strengths OR and SHG have in the metastable phase compared to other materials.

Of particular interest is to see if there is a correlation between the optical rotation and semi-empirical calculations. Although it is possible to calculate OR from first principle using GAUSSIAN [8] in isolated molecules, the only model capable of computing optical rotation in crystals is based on the dipole-dipole interaction of atoms and takes account of long-range Coulomb interactions [9, $10,11,12,13,14,15,16]$. These semi-empirical nonquantum mechanical calculations need electronic polarizability volumes for each atom in the structure as input variables.

A particular limitation of the dipole-dipole interaction model is that it was designed for ionic structures, where less polarizable atoms hardly contribute to the calculated optical properties.

In organic molecules, however, the interactions between atoms are strongly covalent in character. It was found earlier in first-principle calculations that hydrogen atoms in organic molecules cannot be neglected nor is it true that their contributions to optical rotation are small [17]. Hydrogen bonding to aromatic carbons especially was found to be of considerable importance, whereas hydrogen bonding to non-aromatic carbon atoms still may almost be neglected. The 4-MBP molecule consists of two phenyl groups and a methyl group which provides an opportunity to study the difference between hydrogen atoms bonded to a differently hybridized carbon.

We need also to establish a link between the absolute configuration and the sign of optical rotation. Once the relation between optical rotation and absolute structure is found, the optical rotation measured along the optical axis c of 4-MBP can then always be used to determine the absolute structure. Finding the absolute configuration requires special techniques.

Since the basic general form $\{h k i l\}$ does not occur, enantiomorphic (left- and right-handed) crystals cannot be distinguished by morphology.

There are no strong anomalously scattering atoms in 4MBP, thus analysing Bijvoet differences is inconclusive. Instead, we have used the three-beam X-ray technique to determine the actual hand of the screw axis of the same sample on which optical measurements were performed. We shall also provide here a qualitative study of the frequency doubling efficiency ( $d$-coefficients) of 4-MBP. The $d$-coefficients can be calculated from the same theory as for optical rotation [16].

To find the polarizability-volumes one requires knowledge of the refractive indices of 4-MBP which have been determined earlier [1,2], Table 1 . 
Table 1. Refractive indices of uniaxial optically negative metastable 4-methyl benzophenone [1].

\begin{tabular}{l|ll} 
wavelength $(\mathrm{nm})$ & ne & no \\
\hline 535 & 1.5685 & 1.725 \\
589 & 1,5629 & 1.717 \\
671 & 1.5564 & 1.7067
\end{tabular}

4-MBP exhibits a large birefringence, as is observed in many organic crystals. The dipole-dipole interaction theory is usually unable to model such values of birefringence if the hydrogen contribution is neglected, and so we discuss below how this can be overcome.

\section{Methodology}

\section{Absolute configuration}

The absolute configuration of 4-MBP was determined by the experimental determination of triplet phases using three-beam diffraction $[18,19]$. During such an experiment a primary reflection $\mathbf{h}$ is brought into its diffraction position. Afterwards an additional secondary reflection $\mathbf{g}$ is excited by an accurate $\Psi$-azimuthal rotation about the reciprocal lattice vector $\mathbf{h}$. In such a situation the wave fields due to $\mathbf{h}$ and $\mathbf{g}$ interfere with each other by reflection $\pm(\mathbf{h}-\mathbf{g})$. The characteristic intensity change of reflection $\mathbf{h}$ during the $\Psi$-scan of $\mathbf{g}$ through the Ewald sphere depends directly on the triplet phase $\Phi_{3}=\phi(\mathbf{g})+\phi(\mathbf{h}-\mathbf{g})-\phi(\mathbf{h})$. Different absolute structures show opposite signs of the triplet phase. Triplet phases with $\left|\Phi_{3}\right|$ close to $90^{\circ}$ are best suited for the determination of the absolute structure because the difference of the interference profiles is maximum in this case $[18,19]$. Since the signal for the determination of the absolute structure depends directly on the triplet phase, this method is totally independent of anomalous dispersion effects and allows the determination of the absolute structure of an organic compound with very low $\mathrm{O}$ - or $\mathrm{N}$-content and no other strong scattering atoms. This is exactly the situation in the 4-MBP molecule.

\section{Optical Rotation (OR)}

OR measurements in a birefringent crystal sections, requisite to obtain the full tensor, require a careful analysis of the state of polarization of light throughout its passage through the sample. The procedure is described in detail elsewhere, see for a summary Kaminsky, 2000 [20] and most recently, Herreros-Cedres et al. 2005 [21]. Welldefined linearly polarized light in oblique incidence at 
varying angles is analysed with a second polarizer behind the sample (tilter method). The intensity then depends on the geometrical parameters [incidence angle (tilt angle $\alpha$ ), angles of incident polarization and that of the analyzer, and resultant azimuth and ellipticity of the elliptically polarized wave as well as sample extinction direction. The material is described by the optical indicatrix with refractive indices $n e$ and $n o$ and OR, with non-vanishing tensor coefficients $\rho_{11}$ $=\rho_{22}, \rho_{33}$. The coefficient $\rho_{33}$ is obtained directly from a measurement along the optical axis $\mathbf{c}$ of 4-MBP; the others require a sample plate with plate-normal perpendicular to $\mathbf{c}$. The effect of birefringence is obtained from a precise determination of the phase factor $\delta=2 \pi L[n(\alpha)-n o] / \lambda$ of the sample; here $\mathrm{L}$ is sample thickness and $\lambda$ the wavelength, $n(\alpha)$ is the refractive index perpendicular to the incident polarization, no is the refractive index along the incident polarization parallel to the tilt axis. A non-linear leastsquares procedure finds the principal refractive indices from the functional dependence of the trigonometric representations of the various terms contributing to the observed intensity after the sample, i.e. the functional dependence of $\cos (\delta)$. The optical rotation, after successful elimination of spurious and geometrical contributions is given by the so-called 'ellipticity' $\varphi=\varphi_{o} \sin (\delta) / \delta$, where $\varphi_{o}$ is the optical rotation along the path of the light wave.

\section{Second harmonic generation}

Phase-matchability and a qualitative assessment of second harmonic generation was studied on an automated twocircle goniometer attached to a pulsed YAG-laser $(1064 \mathrm{~nm})$ in a set-up in the Clarendon Laboratories of the Department of Physics, Univ. Oxford, UK [22].

\section{Experimental results}

\section{Sample preparation}

4-Methyl benzophenone (Merck) was purified by recrystallization from ethanol. Metastable crystals were grown in the melt supercooled by $0.2-0.5^{\circ} \mathrm{C}$ below the metastable melting point $\left(55^{\circ} \mathrm{C}\right)$ from seed crystals nucleated from highly supercooled melt $[3,5]$. Optically homogeneous crystals of up to $20 \times 20 \times 50 \mathrm{~mm}^{3}$ were obtained within growth periods of $2-7$ days.

Samples for optical measurements were cut with a lapping saw avoiding solvents to prevent transformation into the stable polymorph. Crystals were ground and polished in oil and lapping powder of different grain sizes until optical quality was achieved. 


\section{Absolute Structure}
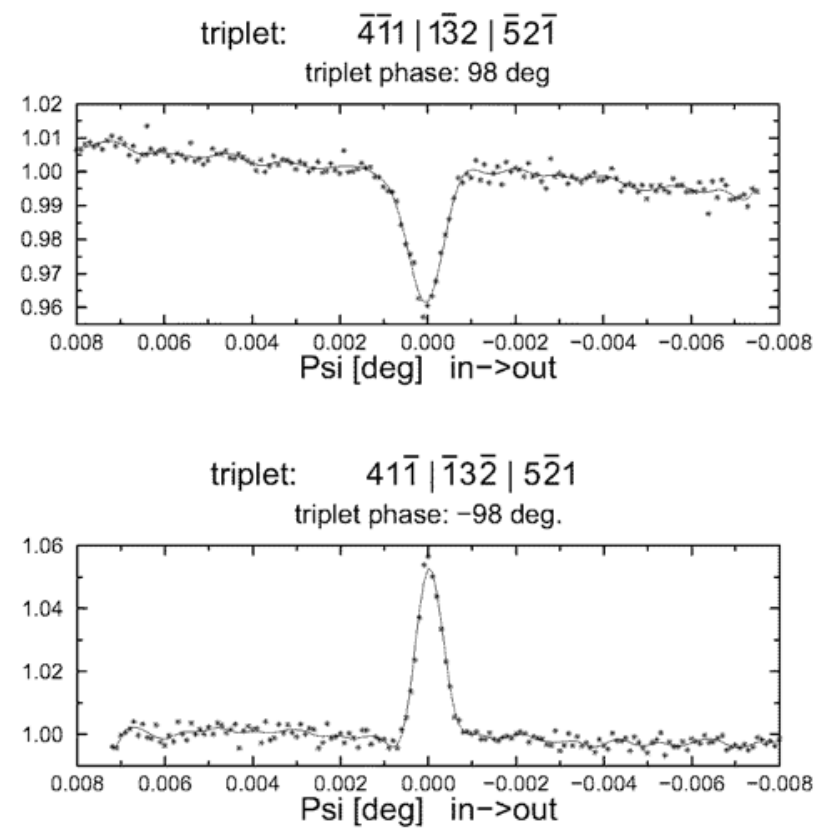

Fig. 2. Experimental three-beam interference profiles for the three-beam case $\overline{\mathbf{4}} 1 \mid \overline{\mathbf{B}} 2 \overline{\mathrm{FR}}$ (top) with $\Phi_{3}=+98^{\circ}$ and its centrosymmetrically related profile $4 \overline{1}|\overline{12}| \overline{2} 1$ (bottom) with $\Phi_{3}=$ $-98^{\circ}$. The experimental profiles agree exactly with the profile forms expected for these triplet phases. The reciprocal lattice vector $\mathbf{g}$ is inside the Ewald sphere for $\Psi>0$ (left side).

Experimental conditions: beamline ID22 (ESRF), Si-(111) double crystal monochromator, $\lambda=0.542 \AA$, $\pi$-polarized radiation.

Three triplet phases were measured for the determination of the absolute structure of 4-MBP, all indicating unambiguously the same space group: $P 3_{1}$. The three beam interference profiles for one three-beam experiment is shown in Figure 2 as a typical example. The destructive interference for the triplet with $\Phi_{3}=+98^{\circ}$ and the constructive interference with $\Phi_{3}=-98^{\circ}$ is clearly visible indicating the correct absolute structure. The three-beam interference experiments were carried out at beamline ID22 at $\operatorname{ESRF}$ (Grenoble).

The structure of 4-MBP then was completely redetermined, with improved positional accuracy of the atomic positions. The crystallographic data can be obtained free of charge from the corresponding author.

\section{Optical rotation}

Only along the optic axis is optical rotation of 4-MBP not affected by birefringence. To find this direction, a sample cut and polished on (0001) was inserted into the tilterdevice [23] and the angle of incidence was changed through tilting the sample within $\pm 20^{\circ}$. Figure 3 shows the result of such a measurement of the azimuth rotation (ellipticity) of 
the elliptically polarized light wave after the sample

(sample thickness: $0.355 \mathrm{~mm}$ ).

Similarly, a sample was prepared that contained the c-axis (optic axis) and the wave vector was tilted towards c.

Figure 4 presents the spectrum derived in a (1 $\overline{\mathbf{0}} 0)$-cut sample of $0.195 \mathrm{~mm}$ thickness.

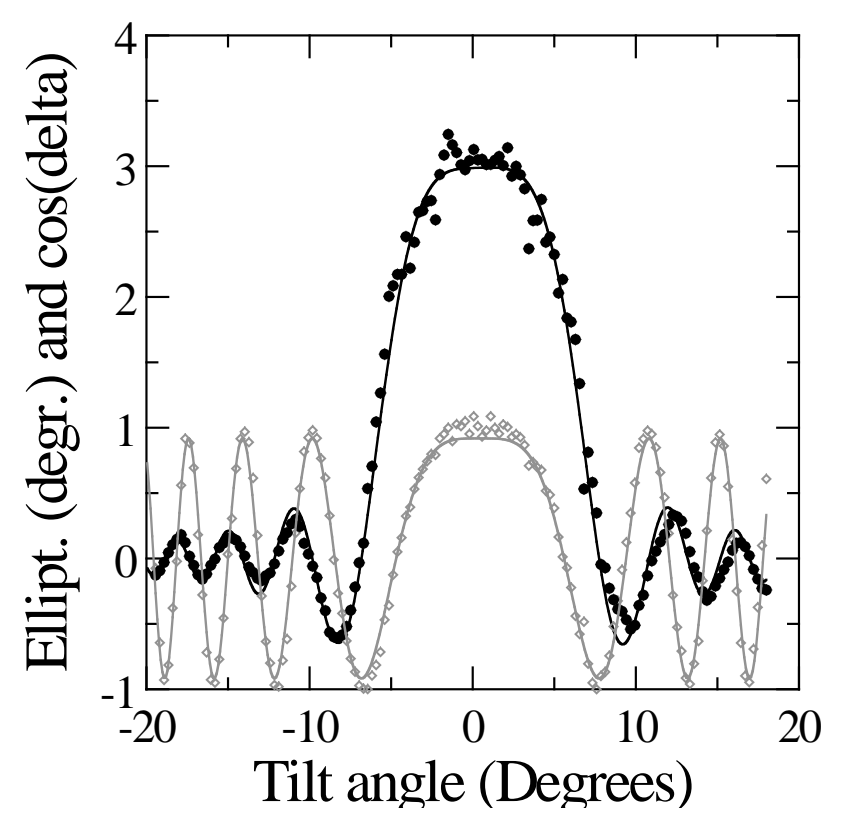

Fig. 3. Azimuthal rotation (ellipticity, black data points) and the $\cos \delta$ (grey data points) of a (0001)-cut 4-MBP sample. Sample thickness $0.355 \mathrm{~mm}$. $\rho_{33}=8.3(7)^{\circ} / \mathrm{mm}$, wavelength $\lambda=670 \mathrm{~nm}$.

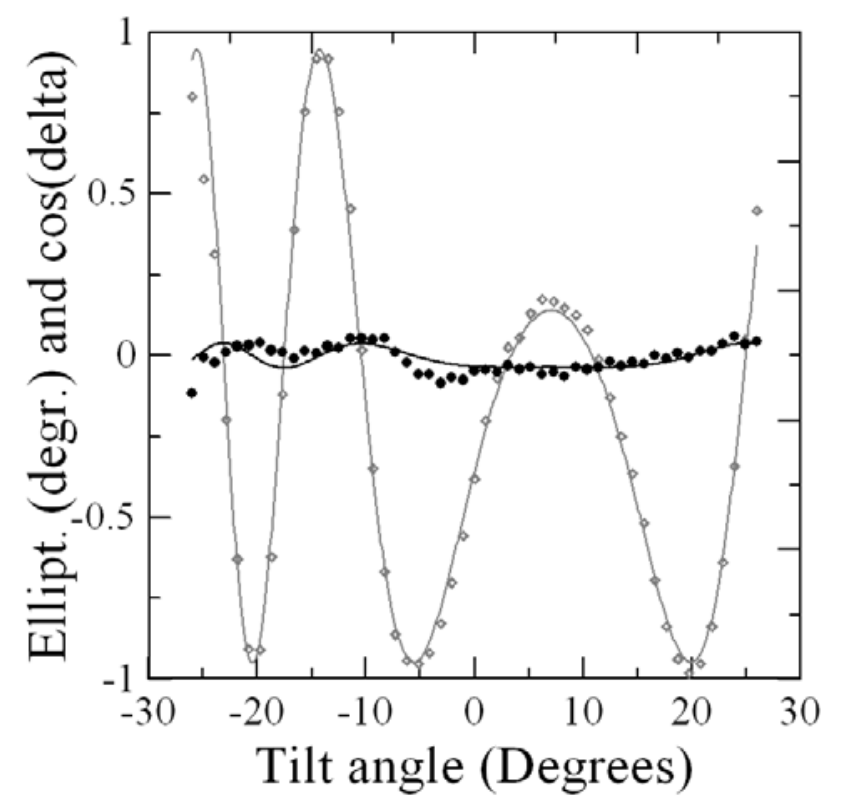

Fig. 4. Azimuthal rotation (ellipticity, black data points) and the

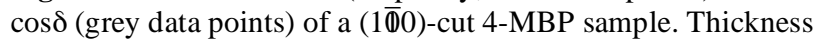
$0.195 \mathrm{~mm} . \rho_{11}=26(5)^{\circ} / \mathrm{mm}, \lambda=670 \mathrm{~nm}$.

The value for $\rho_{11}$ was confirmed with a scan where the sample was rotated by 90 degrees [24]. The result of these 
three experiments is $(\lambda=670 \mathrm{~nm}): \rho_{11}=26(7)^{\circ} / \mathrm{mm}, \rho_{33}=$ $8.3(5)^{\circ} / \mathrm{mm}$. Both rotations are positive, i.e. clockwise if looking towards the light beam.

\section{Qualitative second-harmonic generation study}

A thin (0001) sample plate, thickness $0.2 \mathrm{~mm}$, was mounted on the 'Euler-Wiege' circle $\varphi$ of a two-circle goniometer. The 'Euler-Wiege' circle was rotated around the vertical axis by the goniometer circle angle $v$. Circularly polarized radiation from a Nd:YAG laser $(1064 \mathrm{~nm})$ was focused on the sample and the intensity of the doubled frequency light $(532 \mathrm{~nm})$ was recorded with a photo-multiplier. The result for the accessible range of $-60^{\circ}<\varphi<60^{\circ},-60^{\circ}<v<60^{\circ}$ is shown in Figure 5. The intensities were similar to those of $\mathrm{KH}_{2} \mathrm{PO}_{4}$. However, $\mathrm{SHG}$ measurements require extremely well prepared optical surfaces to allow quantitative analysis, which we were not able to achieve in 4-MBP. Thus, our observations can only confirm the presence of phase-matchability at plate tilts of $v$ of $\pm 56(2)^{\circ}$ where the intensity of the pulses had still be kept to a minimum to avoid spontaneous transformation into the stable polymorph. At present it is unclear if the increased intensities at plate tilts of $\pm 18(2)^{\circ}$ are related to phase matching as well.

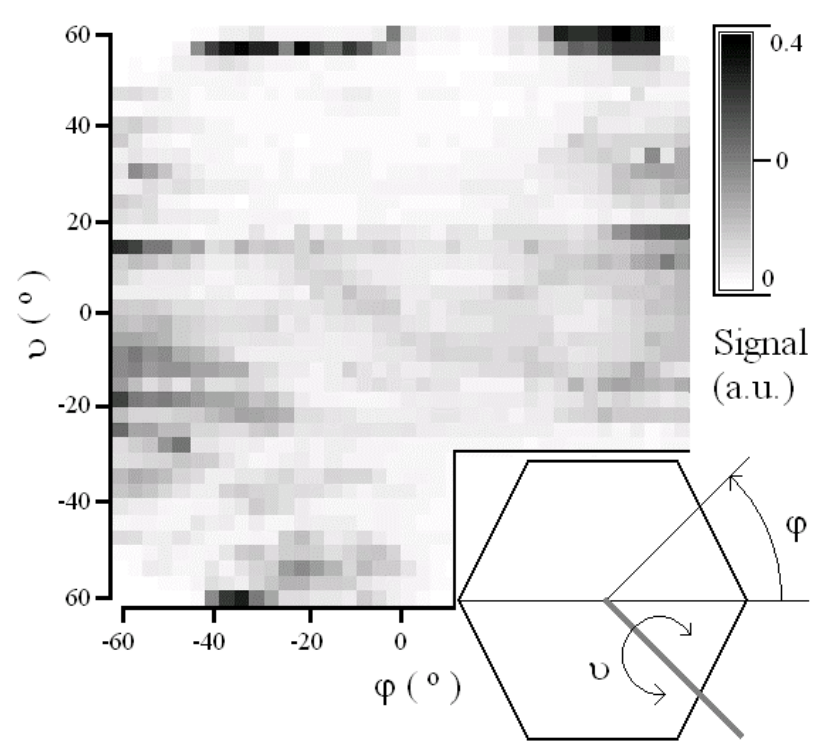

Figure 5. (0001) sample plate, thickness $0.2 \mathrm{~mm}$. Vertical: Makerfringe angle $v$. Horizontal: rotation $\varphi$ of the sample about its normal vector. The colour-shades varying from white to black represent increasing SHG-intensities at initial radiation of $1064 \mathrm{~nm}$. Some angles at the bottom right were not accessible. The insert shows the trigonal prisms, the azimuthal rotation $\varphi$ and the Maker-fringe angle $v$. 


\section{Discussion}

The number of completely determined tensors for optical rotation is still very limited and only a few have been correlated with the absolute structures [20]. Compared with those already known, 4-MBP exhibits average values. Thus we can say that being in a metastable state will not necessarily induce a large OR.

Both tensor components carry the same sign in 4-MBP with SG $P 3_{1}: \rho_{11}=\rho_{22}=26(7)^{\circ} / \mathrm{mm}, \rho_{33}=8.3(5)^{\circ} / \mathrm{mm}(\lambda=$ $670 \mathrm{~nm})$. Low-quartz on the other side has different signs between the components for optical rotation and $\rho_{33}$ with $19.1 \% \mathrm{~mm}$ at $633 \mathrm{~nm}$ is about -2 times larger than $\rho_{11}$, contrary to 4-MBP. From a comparision of completely determined optical rotation tensors of uniaxial crystals [20] one can see that an earlier observation where $\rho_{33} / \rho_{11}$ sometimes is close to $-2 c / a$ [25] with $c$ and $a$ lengths of crystallographic axes $\mathbf{c}$ and $\mathbf{a}$, can not be generalized. In 4MBP $\rho_{33} / \rho_{11}=+0.32$ whereas $-2 c / a=-2.46$.

The question arises if it is possible to predict optical rotation in 4-MBP by the dipole-dipole theory. This would show that the theory is stable enough to describe optical properties even of a metastable compound. We selected polarizability volumes for $\mathrm{O}, \mathrm{C}, \mathrm{H}_{\text {aromatic }}, \mathrm{H}_{\text {methyl }}$ from comparison of calculated- to known refractive indices. Table 2 summarises the results. We used a two-dimensional Newton-Kantorovich algorithm [26] to objectively find a best suited set of polarizabilities for given values of $\mathrm{H}_{\text {methyl }}$ and $\mathrm{O}$.

Table 2. Sets of electronic polarizabilities $\left(\AA^{3}\right)$ for calculating optical properties with the dipole-dipole theory at a wavelength of $670 \mathrm{~nm}$ for oxygen $(\mathrm{O})$, carbon $(\mathrm{C})$, the hydrogen atoms on the phenyl groups $\left(\mathrm{H}_{\text {aromatic }}\right)$, the hydrogen atoms on the methyl group $\left(H_{\text {methyl }}\right) \cdot \rho_{11}, \rho_{33}$ coefficients of optical rotation; $n_{o}, n_{e}$ ordinary and extraordinary refractive indices.

\begin{tabular}{l|llllllll}
\hline$\#$ & $O$ & $C$ & $H_{\text {arom. }}$ & $H_{\mathrm{m} .}$ & $n o$ & $n e$ & $\rho_{11}$ & $\rho_{33}$ \\
\hline 1 & 2.9 & 0.09 & 1.1945 & 0.2 & 1.708 & 1.557 & 9.53 & -11.1 \\
2 & 2.9 & 0.092 & 1.1698 & 0.3 & 1.706 & 1.556 & 8.11 & -5.14 \\
3 & 2.9 & 0.095 & 1.1378 & 0.4 & 1.706 & 1.556 & 6.11 & 1.73 \\
4 & 2.9 & 1.006 & 1.0811 & 0.5 & 1.706 & 1.556 & 1.96 & 12.7 \\
5 & 3 & 0.091 & 1.1773 & 0.2 & 1.706 & 1.556 & 9.14 & -4.09 \\
6 & 3 & 0.094 & 1.1447 & 0.3 & 1.707 & 1.554 & 7.03 & 3.42 \\
7 & 3 & 0.095 & 1.1234 & 0.4 & 1.706 & 1.556 & 5.51 & 8.64 \\
8 & 3 & 1.007 & 1.0699 & 0.5 & 1.708 & 1.557 & 1.14 & 19.9 \\
9 & 3.1 & 0.091 & 1.1625 & 0.2 & 1.707 & 1.557 & 8.97 & 2.97 \\
$\mathbf{1 0}$ & $\mathbf{3 . 1}$ & $\mathbf{0 . 0 9 3}$ & $\mathbf{1 . 1 4 0 7}$ & $\mathbf{0 . 3}$ & $\mathbf{1 . 7 0 6}$ & $\mathbf{1 . 5 5 6}$ & $\mathbf{7 . 4 5}$ & $\mathbf{8 . 5 6}$ \\
11 & 3.1 & 0.095 & 1.1105 & 0.4 & 1.706 & 1.556 & 5.20 & 15.4 \\
12 & 3.1 & 0.100 & 1.0596 & 0.5 & 1.707 & 1.557 & 0.70 & 26.5 \\
\hline
\end{tabular}

The set of parameters best describing the experimental findings is set \# 10 in Table 2 . The value of $\rho_{11}$ in our calculations is always positive for space group $P 3_{1}$ in 
accordance with the experimental findings, although the calculated value is considerably smaller.

The $d$-coefficients for SHG were calculated as outlined earlier [16]. The result is shown below in Table 3, where indeed similar values were found as for $\mathrm{KH}_{2} \mathrm{PO}_{4}$ [22]. The Cartesian reference system $(\mathbf{x}, \mathbf{y}, \mathbf{z})$ is assigned as follows: $\mathbf{z}$ $/ / \mathbf{c}, \mathbf{y} / / \mathbf{b}^{*}, \mathbf{x} / / \mathbf{a}$.

In addition, the normalized coefficients of the electronic contribution to the (clamped) electro-optic effect and electrogyration were calculated, setting all dielectric constants to 1 . Again, these findings compare well with the normalized tensor coefficient in $\mathrm{KH}_{2} \mathrm{PO}_{4}[20$,27].

Representation surfaces were drawn $[28,29]$ in Figure 6 to help in visualizing the tensorial properties.

Table 3. Parameters for the calculation of non-linear optical properties as well as calculated $d$-coefficients $\left(\mathrm{d}_{\mathrm{ijk}} ; 1064 \mathrm{~nm}\right)$, calculated normalized coefficients of the electro-optic effect $\left(r_{i j k}^{\prime}=\right.$ $\mathrm{r}_{\mathrm{ijk}} / \varepsilon_{\mathrm{ij}}$ where $\varepsilon_{\mathrm{ij}}$ are relative dielectric constants; $670 \mathrm{~nm}$ electronic contribution, clamped), and calculated normalized coefficients of electrogyration $\left(\mathrm{g}_{\mathrm{ijk}}^{\mathrm{ijk}}=\mathrm{g}_{\mathrm{ijk}} / \varepsilon_{\mathrm{ij}} ; 670 \mathrm{~nm}\right.$, electronic contribution, clamped).

\begin{tabular}{llll}
\hline Oxygen polarisab. & $3.10 \AA^{3}$ & $\mathrm{~d}_{111}$ & $0.60 \mathrm{pm} / \mathrm{V}$ \\
Carbon polarisab. & $0.0927 \AA^{3}$ & $\mathrm{~d}_{222}$ & $-0.61 \mathrm{pm} / \mathrm{V}$ \\
$\mathrm{H}_{\text {aromatic }}$ polarisab. & $1.1407 \AA^{3}$ & $\mathrm{~d}_{113}$ & $-0.52 \mathrm{pm} / \mathrm{V}$ \\
$\mathrm{H}_{\text {methyl }}$ polarisab. & $0.3 \AA^{3}$ & $\mathrm{~d}_{123}$ & $0.04 \mathrm{pm} / \mathrm{V}$ \\
$\mathrm{n}_{\mathrm{o}}$ (exp.: 1.7067) & 1.7061 & $\mathrm{~d}_{311}$ & $-0.35 \mathrm{pm} / \mathrm{V}$ \\
$\mathrm{n}_{\mathrm{e}}$ (exp.: 1.5564) & 1.5563 & $\mathrm{~d}_{333}$ & $0.45 \mathrm{pm} / \mathrm{V}$ \\
$\mathrm{r}_{111}^{\prime}$ & $-0.1 \mathrm{pm} / \mathrm{V}$ & $\mathrm{g}_{111}^{\prime}$ & $-0.7 \mu^{\circ} / \mathrm{V}$ \\
$\mathrm{r}_{222}^{\prime}$ & $2.20 \mathrm{pm} / \mathrm{V}$ & $\mathrm{g}_{222}^{\prime}$ & $0.7 \mu^{\circ} / \mathrm{V}$ \\
$\mathrm{r}_{113}^{\prime}$ & $3.4 \mathrm{pm} / \mathrm{V}$ & $\mathrm{g}_{113}^{\prime}$ & $-0.1 \mu^{\circ} / \mathrm{V}$ \\
$\mathrm{r}_{131}^{\prime}$ & $1.3 \mathrm{pm} / \mathrm{V}$ & $\mathrm{g}_{131}^{\prime}$ & $0.7 \mu^{\circ} / \mathrm{V}$ \\
$\mathrm{r}_{132}^{\prime}$ & $1.0 \mathrm{pm} / \mathrm{V}$ & $\mathrm{g}_{132}^{\prime}$ & $3.5 \mu^{\circ} / \mathrm{V}$ \\
$\mathrm{r}_{333}^{\prime}$ & $-1.9 \mathrm{pm} / \mathrm{V}$ & $\mathrm{g}_{333}^{\prime}$ & $3.6 \mu^{\circ} / \mathrm{V}$ \\
\hline
\end{tabular}

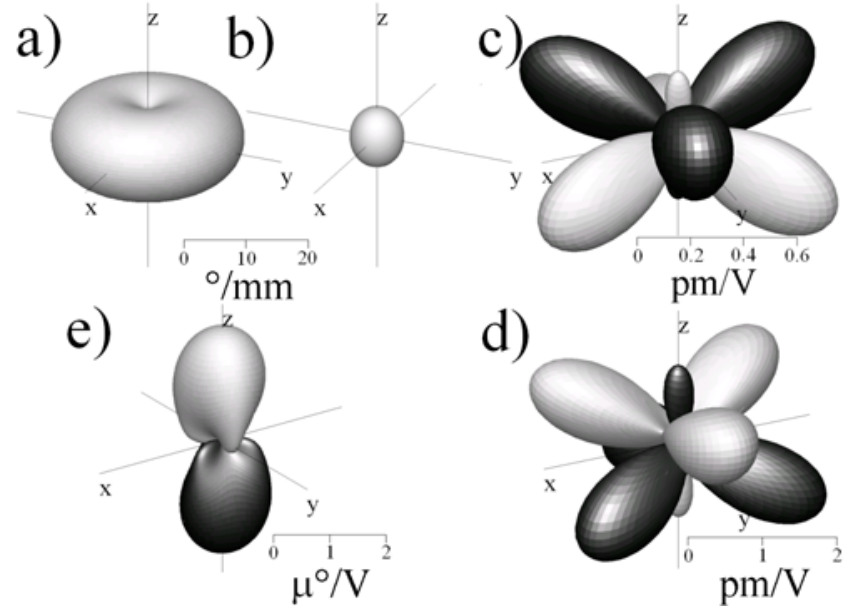

Fig. 6. Representation surface at $670 \mathrm{~nm}$ for a) experimental OR, b) estimated OR; ( $\rho_{33}$ of a) and b) are similar), c) the calculated $d$ coefficients, d) the normalized electro-optic effect (electronic 
contribution, clamped), and e) normalized electrogyration (electronic contribution, clamped). Positive values are shaded in lighter grey, negative values in darker grey tones.

\section{Conclusion}

We can state that it was possible to model reasonably well the experimental findings of refractive indices, optical rotation and second-harmonic generation with the dipoledipole theory. However, the fact that we had to use more than ten times the polarizability for the aromatic hydrogens compared with the polarizability for carbon is clearly of concern in this calculation. This may be an indication of the metastable character of the compound that requires this, and indeed a comparison with other cases indicates that increased hydrogen polarizabilities are necessary to calculate correct refractive indices [30]. However, the case presented here seems to be extreme. The estimated and measured non-linear optical properties are by no means exceptional and are within the range observed in many other materials.

Acknowledgements. We would like to thank M. Drakopoulos for the excellent support at beamline ID22 (ESRF) and R. Mueller and J. Zellner for their support during the three-beam experiments. W.K. is grateful for financial support by the National Science Foundation and the Center for Materials and Devices for Information Technology Research at the University of Washington. We are grateful to S. Haussühl for help with the signassignment of the piezoelectric effect.

\section{References}

[1] Bodewig, C.: Ueber die Dimorphie bei organischen Verbindungen. Poggendorffs Ann. d. Phys. 158 (1876) 232239.

[2] Groth, P.: Chemische Krystallographie, Band V, W. Engelmann, Leipzig, 1919, p. 124.

[3] Kutzke, H.; Al-Mansur, M.; Klapper, H.: Stable and metastable phases of 4-methyl benzophenone. J. Mol. Struct. 374 (1996) 129-135.

[4] Schaum, K.: Über kryptochemischen Polymorphismus. Chemiker-Zeitung 23 (1914) 257-258.

[5] Kutzke, H.: Untersuchungen zur Polymorphie des Benzophenons, des 4-Methylbenzophenons und des Salols. PhD Thesis, Universität Bonn, 2005.

[6] Kaminsky, W.: WinXMorph: A computer program to draw crystal morphology, growth sectors and cross-sections with export files in VRML V2.0 utf8-virtual reality format. J. Appl. Crystallogr. 38 (2005) 566-567.

[7] Haussühl, S.; Kutzke, H.; Klapper, H.: Physical properties of stable monoclinic and metastable trigonal 4-methyl benzophenone. Z. Kristallogr. 205 (2000) 187-189. 
[8] Gaussian03, Pittsburg. http://www.gaussian.com/

[9] Beurskens-Kerssen, G.; Kroon, J.; Endemann, H. J.; van Laar, J.; Bijvoet, J. M.: in Crystallography and Crystal Perfection, ed. G. Ramachandran, Academic Press, London, 1963, p. 225.

[10] Bijvoet, J. M.; Peerdeman, A. F.; Van Bomel, A. J.: Determination of the absolute configuration of optically active compounds by means of X-rays. Nature 168 (1951) 271-272.

[11] Born, M.; Goeppert-Mayer, M.: Dynamische Gittertheorie der Kristalle, in Handbuch der Physik 24 (1933) 623.

[12] Bruhat, G.; Grivet, P.: Rotatoire du quartz pour des rayons perpendiculaires a l'axe optique et sa dispersion dans l'ultraviolet J. Phys. Radium 6 (1935) 12.

[13] van Laar, J.; Endemann, H. J.; Bijvoet, J. M.: Remarks on the relation between microscopic and macroscopic crystal optics. Acta Crystallogr. A 24 (1968) 52-56.

[14] Reijnhart, R.: Classical calculations concerning the double refraction, optical rotation and absolute configuration of Te, $\mathrm{Se}$, Cinnabar (HgS), $\alpha$ - and $\beta$-Quartz, $\beta$-Cristobalite, $\mathrm{NaNO}_{2}$, $\mathrm{NaClO}_{3}$ and $\mathrm{NaBrO}_{3}$. PhD thesis, Delft, 1970.

[15] Devarajan, V.; Glazer, A. M.: Theory and computation of optical rotatory power in inorganic crystals. Acta Cryst. A 42 (1986) 560-569.

[16] Kaminsky, W.; Glazer, A. M.: Crystal optics of mannitol, $\mathrm{C}_{6} \mathrm{H}_{14} \mathrm{O}_{6}$ : Crystal growth, structure, basic physical properties, birefringence, optical activity, Faraday effect, electro-optic related effect and model calculations. Z. Kristallogr. 212 (1997) 283-296.

[17] Kondru, R. K.; Wipf, P.; Beratan, D. N.: Atomic Contributions to the Optical Rotation Angle as a Quantitative Probe of Molecular Chirality. Science 282 (1998) 22472250.

[18] Huemmer, K.; Weckert, E.: Enantiomorphism and the ThreeBeam X-ray Diffraction: Determination of the Absolute Structure. Acta Cryst. A 51 (1995) 431-438.

[19] Weckert, E.; Huemmer, K.: Multiple-Beam X-ray Diffraction for Physical Determination of Reflection Phases and its Application. Acta Cryst. A 53 (1997) 108-143.

[20] Kaminsky, W.: Experimental and phenomenological aspects of circular birefringence and related properties in transparent crystals. Rep. Prog. Phys. 63 (2000) 1575-1640.

[21] Herreros-Cedres, J.; Hernandez-Rodriguez, C.; Kaminsky, W.: Absolute optical rotation of $\mathrm{CsLiB}_{6} \mathrm{O}_{10}$. J. Appl. Crystallogr. 38 (2005) 544-554.

[22] Kaminsky, W.; Fitzmaurice, A. J.; Glazer, A. M.: Measurement and calculation of second-harmonic generation in single-crystal spheres: Application to d-coefficients of Dmannitol, $\mathrm{C}_{6} \mathrm{H}_{14} \mathrm{O}_{6}$. J.Physics D 31 (1998) 767-775.

[23] Kaminsky, W.; Glazer, A. M.: Measurement of optical rotation in crystals. Ferroelectrics 183 (1996) 133-141.

[24] Moxon, J.R.L.; Renshaw, A.R.: The simultaneous measurement of optical rotation and circular dichroism in birefringent linearly dichroic crystal sections: I. Introduction and description of the method. J. Phys.: Condens.Matter, 2 (1990) 6807-6836.

[25] Pine, A.S.; Dresselhaus, G.: Proc. Internat. Enrico Fermi School of Physics, Course LII, ed. Burnstein, Academic, New York, 1971.

[26] Kantorovich, L.V.; Akilov, G.P.: Functional Analysis in Normed Spaces, Pergamon Press, Elmsford, New York, 1964.

[27] Bohatý, L.: Crystallographic aspects of the linear electrooptic effect. Z.Kristallogr. 166 (1984) 97-119. 
[28] Kaminsky, W.: Graphische Darstellung von Tensoren: unverzichtbar in der Kristallphysik. DGK-Meeting (1997), Hamburg, Germany. Z. Kristallogr. Suppl. 12 (1997) 183.

[29] Kaminsky, W.: Wintensor: ein WIN95/98/NT Programm zum Darstellen tensorieller Eigenschaften. DGK-Meeting (2000), Aachen, Germany. Z. Kristallogr. Suppl. 17 (2000) 51.

[30] Claborn, K.; Kahr, B.; Kaminsky, W.: Calculation of optical properties of the tetraphenyl-X family of isomorphous crystals (X=C, Si, Ge, Sn, Pb). Cryst. Eng. Comm. 4 (2002) $252-256$ 